\title{
Transfert de chaleur par convection naturelle dans une cavité rectangulaire isotherme ouverte sur une face
}

\author{
F. Penot \\ Laboratoire d'Etudes Aérodynamiques et Thermiques, 40, avenue du Recteur-Pineau, 86022 Poitiers, France
}

(Reçu le 24 juillet 1979, révisé le $1^{\mathrm{er}}$ octobre 1979, accepté le 2 octobre 1979)

\begin{abstract}
Résumé. - L'étude présentée ici concerne la modélisation par le calcul numérique des écoulements de convection naturelle qui se développent au sein d'une cavité rectangulaire, chauffée à température constante et ouverte sur une de ses faces. Pour différents nombres de Grashof $\left(<10^{7}\right)$, on calcule les nombres de Nusselt locaux et globaux de la cavité et les résultats sont confrontés à ceux de la plaque plane verticale mettant en évidence un effet de cavité sur le transfert convectif de chaleur. En outre on caractérise l'influence de l'inclinaison de la cavité et les résultats sont discutés.
\end{abstract}

\begin{abstract}
This paper concerns the modelization of free convection flows inside an isothermal rectangular cavity opened on one side. For different values of Grashof number $\left(<10^{7}\right)$, local and global Nusselt numbers are determined and results are compared with those obtained in the case of the vertical flat plate. A cavity effect is clearly shown. Furthermore the influence of the inclination of the cavity is demonstrated and results are discussed.
\end{abstract}

\section{Nomenclature}

$A, B, a, m, n=$ coefficients du changement de variable.

$C_{\mathrm{p}} \quad=$ chaleur massique à pression constante.

$d \quad=$ demi-ouverture de la cavité.

$g=$ intensité de la pesanteur.

$G r=g \beta\left(T_{1}-T_{0}\right) d^{3} / v^{2}=$ nombre de Grashof.

$k=$ conductivité thermique.

$L \quad=$ allongement de la cavité.

$\mathrm{Nu} \quad=$ nombre de Nusselt local.

$\overline{N u_{i}} \quad=$ nombre de Nusselt de la face $i$.

$\overline{\mathrm{Nu}} \quad=$ nombre de Nusselt global.

$\operatorname{Pr} \quad=$ nombre de Prandtl.

$\bar{q} \quad=$ flux de chaleur.

$q \quad=$ densité de flux de chaleur.

$t \quad=$ temps.

$T \quad=$ température.

$T_{0} \quad=$ température du fluide à l'infini.

$T_{1} \quad=$ température de paroi de la cavité.

$u, v=$ composantes de la vitesse.

$x, y=$ variables d'espace.

$X, Y=$ variables d'espace sans dimension.

$\beta \quad=$ coefficient d'expansion thermique.

$\rho \quad=$ masse volumique du fluide.

$v \quad=$ viscosité cinématique.

$\varphi \quad=$ angle d'inclinaison du plan d'ouverture par rapport à la verticale descendante.

$\psi \quad=$ fonction de courant.

$\omega \quad=$ rotationnel de la vitesse.
1. Introduction. - Avec le développement récent des récepteurs solaires en forme de cavités ouvertes, se pose le problème de leur rendement énergétique. Dans ce type de capteur, les pertes de chaleur sont principalement : les pertes radiatives qui peuvent être appréhendées par le calcul $[1,2]$; les pertes par convection naturelle et forcée qui sont plus difficiles à évaluer.

En l'absence de vent, les parois internes de la cavité portées à des températures élevées induisent des mouvements de convection naturelle de l'air au voisinage des parois. Cette convection est alimentée par l'air frais qui pénètre par l'ouverture.

S'il existe des travaux concernant la convection naturelle dans les cavités fermées [3,4], aucune étude n'a été faite dans le cas des cavités ouvertes, mis à part l'étude de thermosiphons [5, 6], dont les résultats ne peuvent s'appliquer ici, ainsi que la donnée de quelques bilans thermiques approximatifs effectués sur des prototypes de chaudières solaires [7]. Ces auteurs s'accordent sur le fait que les pertes par convection naturelle pourraient être de l'ordre de 2 à $5 \%$ de la puissance incidente.

On présente dans cet article les principaux résultats obtenus par une étude numérique des écoulements de convection naturelle qui apparaissent dans une cavité isotherme ouverte sur le milieu ambiant plus froid. 
2. Equations et méthode numérique. - 2.1 LES ÉQUATIONS DE LA CONVECTION NATURELle. - Cette étude a été réalisée dans le cadre d'hypothèses simplificatrices :

L'écoulement est supposé bidimensionnel, c'est-àdire que l'on néglige l'influence des parois latérales, ce qui n'est vrai en toute rigueur que pour les cavités d'ouvertures très allongées dans la direction horizontale.

On suppose en outre que le fluide satisfait aux hypothèses de Boussinesq : en particulier sa masse volumique est constante sauf lorsque ses variations créent des forces pesantes, les autres propriétés physiques sont constantes.

Les équations de conservation de la masse, de la quantité de mouvement et de l'énergie régissent l'écoulement instationnaire du fluide dans la cavité.

En introduisant la fonction de courant $\psi$ définie par

$$
u=\frac{\partial \psi}{\partial y} ; \quad v=-\frac{\partial \psi}{\partial x}
$$

où $u$ et $v$ désignent les composantes de la vitesse suivant $O x$ et $O y$ (Fig. 1), de même, en introduisant le rotationnel $\omega$ de la vitesse défini par :

$$
\omega=\frac{\partial v}{\partial x}-\frac{\partial u}{\partial y}=-\left(\frac{\partial^{2} \psi}{\partial x^{2}}+\frac{\partial^{2} \psi}{\partial y^{2}}\right)
$$

les équations s'écrivent :

$$
\begin{array}{r}
\frac{\partial \omega}{\partial t}+u \frac{\partial \omega}{\partial x}+v \frac{\partial \omega}{\partial y}=g \beta\left(\frac{\partial T}{\partial y} \cos \varphi-\frac{\partial T}{\partial x} \sin \varphi\right)+ \\
+v\left(\frac{\partial^{2} \omega}{\partial x^{2}}+\frac{\partial^{2} \omega}{\partial y^{2}}\right) \\
\frac{\partial T}{\partial t}+u \frac{\partial T}{\partial x}+v \frac{\partial T}{\partial y}=\frac{k}{\rho C_{\mathrm{P}}}\left(\frac{\partial^{2} T}{\partial x^{2}}+\frac{\partial^{2} T}{\partial y^{2}}\right)
\end{array}
$$

avec $\varphi$ : angle d'inclinaison du plan d'ouverture de la cavité par rapport à la direction de la pesanteur.

2.2 Conditions auX Limites et initiales. - Sur les parois internes de la cavité on exprime des conditions d'adhérence du fluide (vitesse nulle, $\psi=0$ ) et des conditions de température constante : $T=T_{1}$.

Sur la face ouverte, il n'est pas possible d'imposer des conditions aux limites sans influencer irrémédiablement l'écoulement dans la cavité.

Afin de surmonter cette difficulté, on ferme le domaine par des surfaces fictives placées suffisamment loin de la section d'entrée (Fig. 1).

Les conditions aux limites peuvent s'écrire alors :

pour $y= \pm m d$ et $-n L d \leqslant x \leqslant 0: \frac{\partial T}{\partial y}=0, u=0$

pour $x=0$ et $|y| \geqslant d: \frac{\partial T}{\partial x}=0, u=v=0, \psi=0$

pour $x=-n L d: T=T_{0}, v=0$.

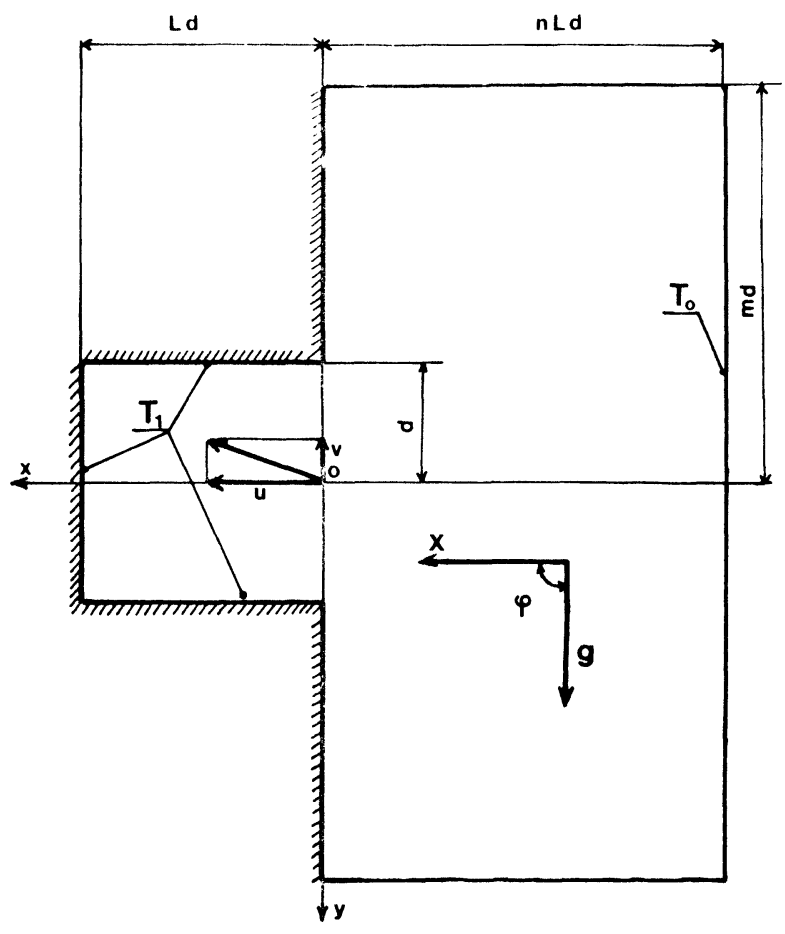

Fig. 1. - Schéma de la cavité.

[Cavity scheme.]

Cette dernière condition reste réaliste aux petites valeurs de $\varphi$ si $n$ est très grand.

Les conditions aux limites sur la fonction de courant se déduisent des éqs. (1), celles sur le rotationnel de la vitesse se déduisent de l'éq. (2).

Au temps $t=0$, on porte subitement les parois de la cavité à la température $T_{1}$, le fluide étant partout au repos et à la température $T_{0}$.

2.3 LA MÉTHODE NUMÉRIQUE. - Le système d'éqs. (1) à (4) ainsi que les conditions aux limites (5) sont rendus adimensionnels en introduisant les grandeurs de référence suivantes :

- $d$ : demi-ouverture de la cavité,

- $\left(T_{1}-T_{0}\right)$ : écart de température,

- $d^{2} / v$ : temps.

Les solutions sont alors fonction de deux paramètres sans dimension :

$\operatorname{Pr}=\rho \nu C_{\mathrm{p}} / k, \quad$ nombre de Prandtl

$G r=g \beta\left(T_{1}-T_{0}\right) d^{3} / v^{2}$, nombre de Grashof .

De plus un changement de variable identique à celui utilisé par Kettleborough [8] :

$$
\begin{aligned}
& x=\operatorname{Lnd}\left[A(X)^{a}+(1-A) X\right] \\
& y=\operatorname{md}\left[B(Y)^{b}+(1-B) Y\right]
\end{aligned}
$$

$L$ étant l'allongement de la cavité, permet d'obtenir une meilleure définition des grandeurs au voisinage de la section d'entrée. 
Le système d'équations transformées est résolu par une méthode aux différences finies.

Pour chaque pas de temps, les équations de la température et du rotationnel de la vitesse sont résolues par une méthode aux directions alternées (ADI). Les termes convectifs du type $u \frac{\partial u}{\partial x}, \ldots$ sont représentés avec une précision du second ordre par des différences décentrées vers l'amont. L'équation de la fonction de courant est résolue par une méthode de relaxation classique [3].

On détermine ensuite les composantes $u$ et $v$ de la vitesse par une méthode explicite directe.

Une extrapolation linéaire des vitesses et de la fonction de courant sur les deux derniers pas de temps permet en général d'obtenir une approximation suffisante pour le calcul de la température et du rotationnel au pas de temps suivant, sinon on engage un processus itératif sur le calcul des grandeurs à ce même pas de temps.

2.4 Nombre De Nusselt. - Le flux de chaleur $\bar{q}$ qui traverse une paroi de longueur $s$ et de largeur unitaire s'écrit :

$$
\bar{q}=k{ }_{0}^{s} \frac{\partial T}{\partial n} \mathrm{~d} s
$$

$\partial / \partial n$ étant la dérivée normale à la paroi.

Dans le cas de la cavité de la figure 1, ce flux peitt s'écrire :

$$
\bar{q}=\sum_{i=1}^{3} \bar{q}_{i}
$$

avec $q_{i}$ flux par unité de largeur qui traverse une face.

On définit le nombre de Nusselt global de la cavité par :

$$
\bar{N} u=\frac{\bar{q}}{k\left(T_{1}-T_{0}\right)}
$$

ainsi que le nombre de Nusselt global d'une face par :

$$
\bar{N} u_{i}=\frac{\bar{q}_{i}}{k\left(T_{1}-T_{0}\right)} .
$$

Le nombre de Nusselt local est défini à partir de la densité de flux de chaleur $q$ à la paroi, soit :

$$
N u=\frac{q d}{k\left(T_{1}-T_{0}\right)} .
$$

Les nombres de Nusselt sont fonction du temps. Ils peuvent donc être définis soit de façon instantanée, soit par une valeur moyenne sur un intervalle de temps fixé à priori.

3. Résultats. - 3.1 Conditions du CALCUl. On présente ici les résultats de calculs effectués sur un maillage de 812 nœuds obtenu à partir de 27 pas d'espace suivant l'axe $O X$ et 28 pas suivant $O Y$, ce qui correspond à 140 mailles utiles à l'intérieur de la cavité. Différents essais ont montré que si la distance entre le plan d'ouverture de la cavité et les frontières extérieures du domaine sont supérieures à $6 \mathrm{~d}$, les champs de vitesse et de température ne sont pas modifiés de façon notable. Il en a été de même en considérant un maillage double du précédent $(56 \times 58)$.

Les calculs ont donc été effectués dans les conditions suivantes : une cavité carrée $(L=2)$ avec $n=3$ et $m=5(6)$, dans le cas de l'air $(P r=0,73)$ pour des valeurs du nombre de Grashof inférieure à $10^{7}$ et des inclinaisons comprises entre 0 et $180^{\circ}$. Au-delà de $G r=10^{7}$ une représentation correcte des couches limites qui se développent le long des parois nécessiterait un maillage trop serré et donc des coûts de calcul prohibitifs.

\subsection{RECHERCHE DE SOLUTIONS STATIONNAIRES. -} Dans le cas où le plan d'ouverture de la cavité est vertical $\left(\varphi=90^{\circ}\right)$, les courbes de la figure 2 montrent que le nombre de Nusselt global de la cavité ne tend plus vers une valeur constante dans le temps lorsque $G r$ est supérieur à $10^{5}$.

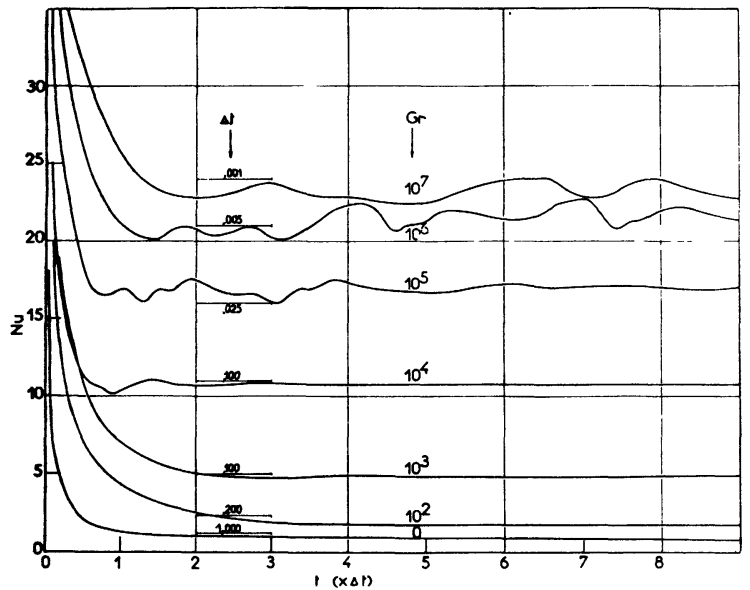

Fig. 2. - Evolution du nombre de Nusselt global en fonction du temps $\left(\varphi=90^{\circ}\right)$.

[Global Nusselt number versus time $\left(\varphi=90^{\circ}\right)$.]

Des instabilités thermiques apparaissent alors le long de la paroi horizontale inférieure.

De même on n'atteint pas de solution stationnaire lorsque le plan d'ouverture de la cavité est dirigé vers le haut $\left(\varphi<90^{\circ}\right)$ (Fig. 3). L'inclinaison de la paroi inférieure induit des écoulements montants qui sont contraires à l'écoulement global dans la cavité et donc source d'instabilités dynamiques (Fig. 4).

Des noyaux de fluide chaud se forment alors le long de cette paroi, puis ils se détachent et sont entraînés dans la cavité.

3. 3 LE NOMBRE DE NusSelt LOCAL. - Les nombres de Nusselt locaux instantanés calculés à partir des profils de température dans la cavité sont représentés 


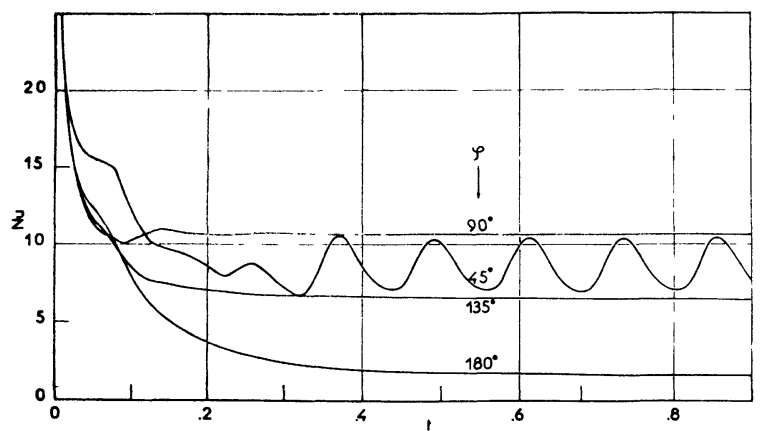

Fig. 3. - Evolution du nombre de Nusselt local en fonction du temps $\left(G r=10^{4}\right)$.

[Global Nusselt number versus time $\left(G r=10^{4}\right)$.]

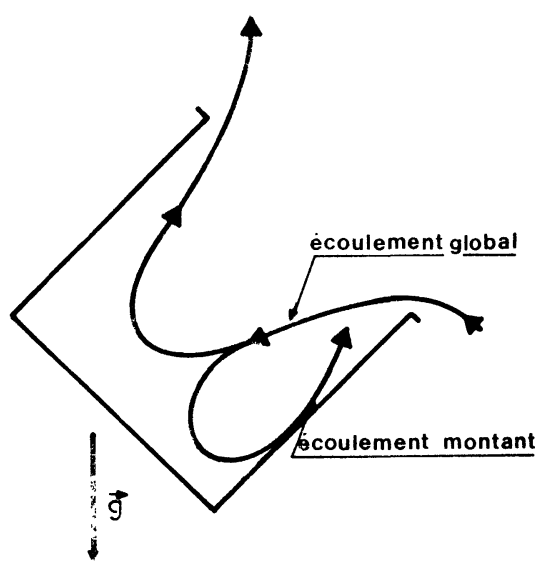

Fig. 4. - Exemple d'écoulement pour $\varphi<90^{\circ}$.

[Flow example at $\varphi<90^{\circ}$.]

figure 5 pour différentes valeurs du nombre de Grashof et figure 6 pour différentes inclinaisons.

On peut noter en particulier à $G r=10^{6}$ et $G r=10^{7}$ (Fig. 5), ainsi qu'à $\varphi=45^{\circ}$ (Fig. 6) la présence d'un creux très marqué dans le profil du nombre de Nusselt local sur la face inférieure (face 3) qui correspond à la formation d'un noyau de fluide chaud. Aux arêtes de la cavité isotherme, il n'y a pas d'échange de chaleur, il en résulte un nombre de Nusselt local nul (Figs. 5, 6).

3.4 Le NOMBRe DE NusSelt SUR UNE FACE. A $\varphi=90^{\circ}$, les transferts de chaleur sur la paroi inférieure sont, en moyenne dans le temps, toujours supérieurs aux transferts sur les deux autres faces (Fig. 7).

Pour une inclinaison de $180^{\circ}$ (cavité tournée vers le bas) un léger mouvement de convection subsiste dans la cavité. Le nombre de Nusselt sur chacune des faces verticales est supérieur à celui obtenu en conduction pure (Fig. 7). En conduction pure, c'est-à-dire pour $G r=0, \bar{N} u_{1}=\bar{N} u_{3}=0,33, \bar{N} u_{2}=0,05$.

Pour des inclinaisons intermédiaires $\left(\varphi=135^{\circ}\right)$ les faibles valeurs du nombre de Nusselt sur les parois

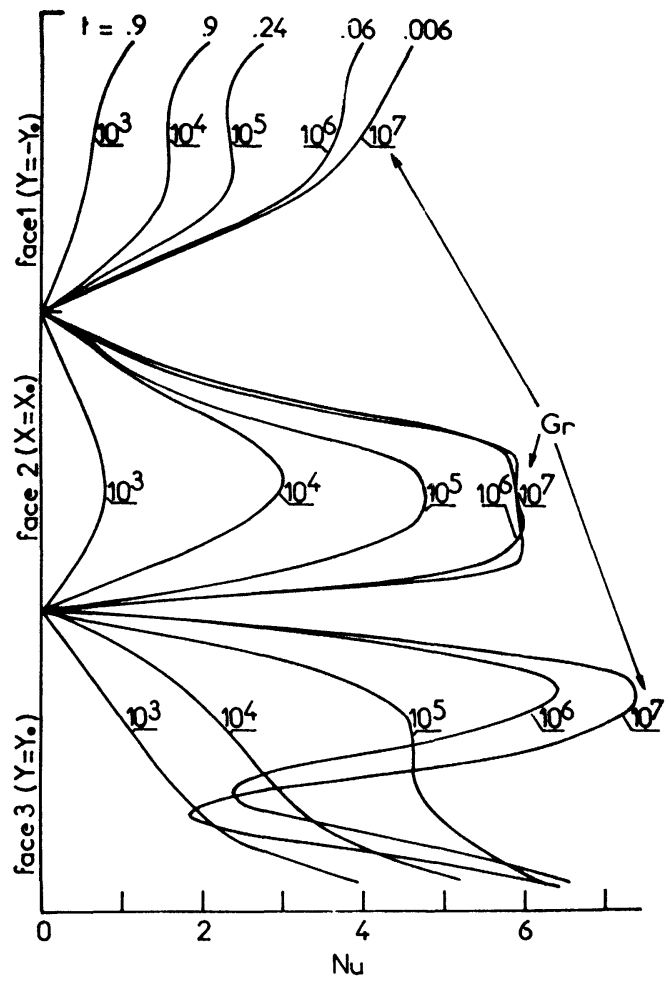

Fig. 5. - Variation du nombre de Nusselt local $\left(\varphi=90^{\circ}\right)$ (face $1=$ paroi supérieure; face $2=$ paroi verticale; face $3=$ paroi inférieure).

[Variation of the local Nusselt number $\left(\varphi=90^{\circ}\right)$ (face $1=$ upper side; face 2 = vertical side; face 3 = lower side).]

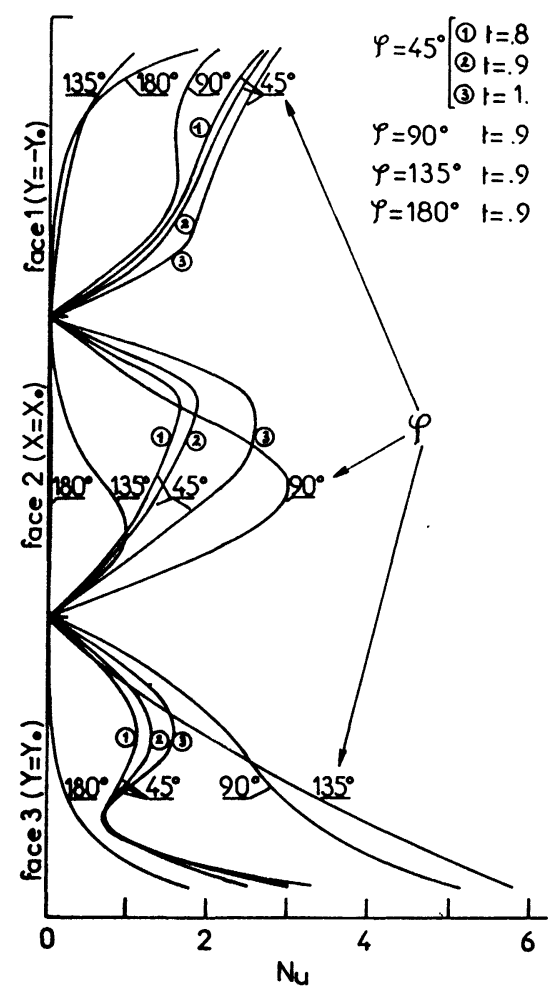

Fig. 6. - Variation du nombre de Nusselt local $\left(G r=10^{4}\right)$.

[Variation of the local Nusselt number $\left(G r=10^{4}\right)$.] 


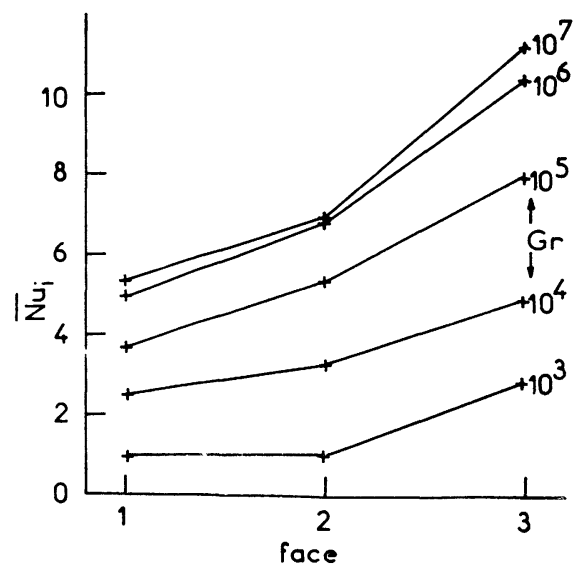

Fig. 7. - Nombre de Nusselt moyen sur chaque face $\left(\varphi=90^{\circ}\right)$.

[Mean Nusselt number on each side $\left(\varphi=90^{\circ}\right)$.]

supérieures 1 et 2 (Fig. 8) et du nombre de Nusselt local (Fig. 6) sont révélatrices de l'existence d'une zone de fluide chaud presque stagnant dans l'angle supérieur de la cavité.

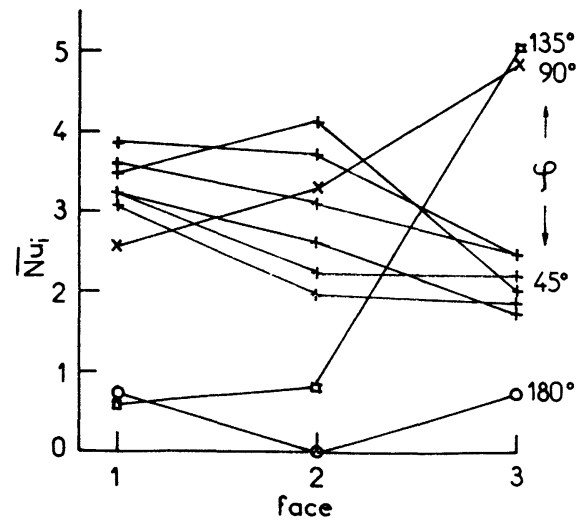

Fig. 8. - Nombre de Nusselt global sur chaque face $\left(G r=10^{4}\right)$ pour $\varphi=90^{\circ}, 135^{\circ}, 180^{\circ}$ : solution stationnaire; pour $\varphi=45^{\circ}$ : à 6 instants différents.

[Global Nusselt number on each side $\left(G r=10^{4}\right)$ when $\varphi=90^{\circ}$, $135^{\circ}, 180^{\circ}$ : steady solution when $\varphi=45^{\circ}$ : at 6 different times.]

3.5 Le NOMBRe De Nusselt GLOBAL, eFFet DE CAVITÉ. - Il est intéressant de comparer les échanges de chaleur par convection dans la cavité à ceux d'une plaque plane verticale en convection naturelle laminaire (Fig. 9).

Quelle que soit la valeur de $G r$, le nombre de Nusselt global moyen de la cavité d'ouverture verticale est toujours inférieur à celui d'une plaque plane verticale chauffée à la même température et d'une hauteur égale à la longueur de la cavité développée.

De même pour $G r \geqslant 5 \times 10^{5}$ les pertes par convection dans la cavité sont plus faibles que celles d'une plaque verticale de hauteur égale à l'ouverture de la cavité. Elles sont supérieures pour $10^{3}<G r<10^{5}$.

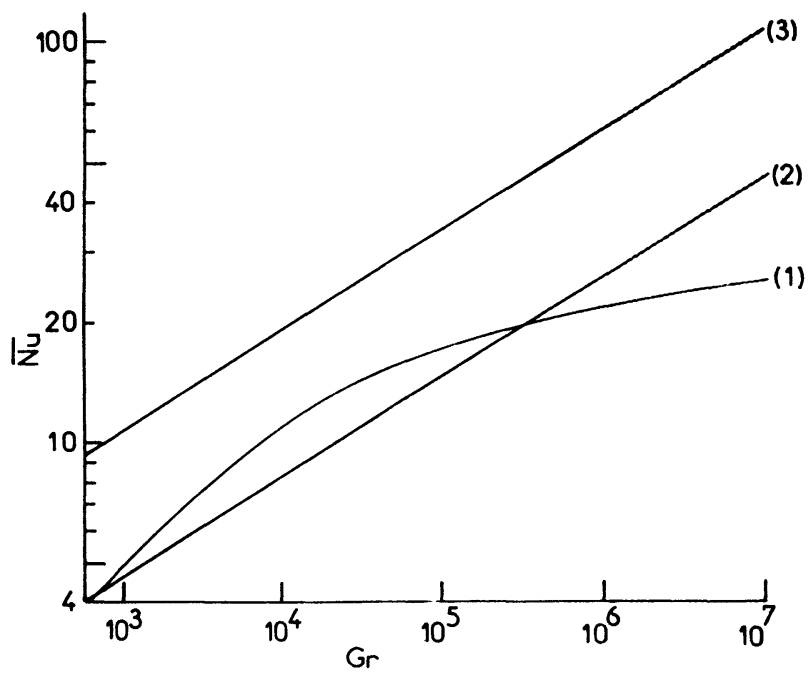

Fig. 9. - Nombre de Nusselt global moyen à $\varphi=90^{\circ}$ comparé aux lois laminaires de la convection naturelle sur les plaques planes verticales d'après [9]. (1) Cavité ouverte. (2) Plaque plane verticale de hauteur 2d. (3) Plaque plane verticale de hauteur 6d.

[Mean global Nusselt number at $\varphi=90^{\circ}$ compared with vertical flat plates in laminar free convection by [9]. (1) Opened cavity; (2) $2 \mathrm{~d}$ height vertical flat plate; (3) $6 \mathrm{~d}$ height vertical flat plate.]

3.6 INFLUENCE DE L'INCLINAISON. - A $G r=10^{4}$, la figure 10 montre les variations du nombre de Nusselt global en fonction de l'angle d'inclinaison du plan d'ouverture. Pour $\varphi \geqslant 90^{\circ}$, on remarque que les points calculés suivent approximativement une loi sinusoïdale, cette loi étant calculée à partir de la valeur maximale de $\bar{N} u$ à $90^{\circ}$ et de sa valeur minimale à $180^{\circ}$.

Les pertes par convection sont donc d'autant moins importantes que l'ouverture de la cavité est orientée vers le bas.

Pour $\varphi<90^{\circ}$ la loi sinusoïdale n'est plus vérifiée,

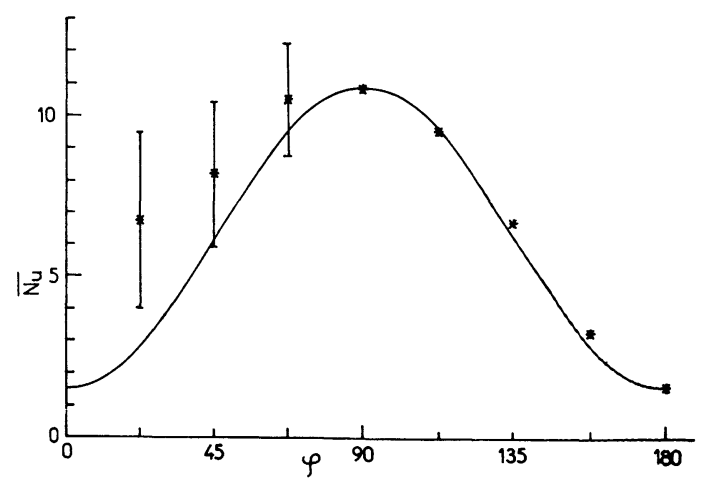

Fig. 10. - Evolution du nombre de Nusselt global en fonction de l'inclinaison $\left(G r=10^{4}\right)$. — Loi sinusoïdale ; * valeur moyenne; I domaine de variation de $\mathrm{Nu}$ lorsque la solution n'est pas stationnaire.

[The global Nusselt number versus inclination $\left(G r=10^{4}\right)$. nusoidal law; * mean value; $F$ range of variation when no steady solution exists.] 
les problèmes posés pour deux inclinaisons symétriques par rapport à $90^{\circ}$ n'étant pas les mêmes.

4. Conclusion. - Cette étude, bien qu'incomplète $\mathrm{du}$ fait du caractère bidimensionnel utilisé et de la limitation du calcul à des nombres de Grashof rela- tivement faibles, a toutefois permis de mettre en évidence un phénomène caractéristique de ce type d'écoulement qui est l'effet de cavité. Il en résulte que les formules classiques d'ingénieur ne peuvent s'appliquer à ce problème sans introduire d'erreur appréciable.

\section{Bibliographie}

[1] Bernard, J., Contribution à l'étude des cavités rayonnantes et des chaudières solaires. Thèse de docteur-ingénieur (Poitiers) Dec. 1977.

[2] Blay, D., Haziza, C., Analitycal and experimental determination of radiation and temperature distribution inside solar receivers (Atlanta-Georgia) ISEC 1979.

[3] Wilkes, CHurChILl, The finite computation of natural convection in a rectangular enclosure, AICHe Journal 12, $\mathrm{n}^{\circ} 1$ (1966) 161-166.

[4] Jannot, M., Mazeas, C., Etude expérimentale de la convection naturelle dans les cellules rectangulaires verticales, Int. J. Heat Mass Transfer. 16 (1973) 81-100.
[5] JAPIKSE, Advances in thermosyphon technology Academic Press, Adv. Heat Transfer 9 (1973) 2.106.

[6] Penot, F., Sur le fonctionnement d'un thermosiphon résultant de la rupture d'équilibre instable de fluide stratifié, A paraître.

[7] Proceedings of US, French meeting on convective losses of solar plants (Paris) 1978.

[8] Kettleborough, C. F., Transient laminar free convection between heated vertical plates including entrance effects, Int. J. Heat Mass Transfer 15 (1972) 883-896.

[9] GiBlin, Transmission de la chaleur par convection naturelle. Collection de l'ANRT (Eyrolles Paris) 1974 p. 41. 